\title{
Introducing clinical protocol for ultrasound-guided high-intensity focused ultrasound ablation of uterine fibroids in patients in Europe, provided from experienced Chinese center-prospective comparative.
}

\author{
Dobromir Dimitrov ${ }^{1}$, Kun Zhou ${ }^{2 *}$, Martin Karamanliev', Joana Joncheva1, Wei Yang ${ }^{2}$, Lifeng Ran², \\ Grigor Gorchev ${ }^{1}$, Chengbing Jin², Slavcho Tomov', Ventsislav Georgiev ${ }^{1}$, Jun Zhang², Yuhong Ma², \\ Hui $\mathrm{Zhu}^{2}$, Nadya Stanislavova ${ }^{1}$ \\ ${ }^{1}$ Medical University-Pleven, 5800, Bulgaria \\ ${ }^{2}$ Clinical Center for Tumor Therapy, the Second Affiliated Hospital, Chongqing Medical University, Chongqing, \\ 400010, PR China
}

\begin{abstract}
Introduction: This study aims to determine whether the Chinese clinical protocol, ultrasound guided high-intensity focused ultrasound (USgHIFU) combined with real-time monitoring using a contrast enhanced ultrasound (CEUS), also applies well in European patients with uterine fibroids.

Methods: Seventy-five patients with 87 nodules of uterine fibroids from China and Bulgaria were treated with USgHIFU from April 2015 to July 2016, in the study. In Group A (Bulgarian) 26 (33 nodules) patients and in group B (Chinese) 49 (54 nodules) patients were included in the study. The same clinical procedure of USgHIFU treatment as well as a follow-up was performed on patients from China and Bulgaria. The general characteristics of the patients, the USgHIFU treatment and evaluation parameters, including; complications, fibroid size, tumor volume, subcutaneous fat size, non-perfused volume (NPV) and shrinkage process, were comparatively studied in Chinese and Bulgarian patients. Results: There were no significant differences in clinical parameters of Chinese and Bulgarian patients with uterine fibroids, such as vertical, left-right and anterior-posterior size, treatment time, USgHIFU time. After USgHIFU treatment, no complications were recorded in both groups. On the $6^{\text {th }}$ and $12^{\text {th }}$ month after USgHIFU, fibroid nodules shrunk and the NPV was statistically significant compared to NPV before USgHIFU, but there were no statistically significant differences.

Conclusions: The initial results of our study showed that CEUS clinical protocol for real time monitoring of efficacy and quality of the USgHIFU for uterine fibroid treatment that had been used in Chinese centers may also be applicable for European patients.
\end{abstract}

Keywords: Ultrasound guided High-Intensity Focused Ultrasound (USgHIFU), Contrast enhanced ultrasonography (CEUS), Real-time monitoring, Uterine fibroid.

Accepted on September 22, 2018

\section{Introduction}

Uterine fibroid (leiomyoma, myoma) is the most common benign gynaecological tumor. Mostly it can be found in women aged 30-50 years, but rarely occurs in younger women (20-35 years). Fibroids appear more often in black women (up to $80 \%$ ), than in white women (20-30\%) [1]. Uterine fibroids can be classified by their location (subserosal, intramural and submucosal), by their quantity (single or multiple), by size or perfusion.

Traditional treatment of uterine fibroids is based on symptoms, size and location of the fibroids, and the woman's desire for fertility. Treatment methods include medical management (both hormonal and nonhormonal), selective uterine artery embolization (UAE), HIFU, radiofrequency ablation, and surgical alternatives including myomectomy (abdominal, hysteroscopic, vaginal and laparoscopic/robotically assisted), hysterectomy (abdominal, vaginal and laparoscopic/robotically assisted), myolysis (bipolar, cryo, radiofrequency, laparoscopic, and MRI-guided laser) and uterine artery ligation [2,3]. Non-invasive methods (UAE, HIFU and radiofrequency ablation) are increasingly being applied in practice.

The Society of Obstetricians and Gynaecologists of Canada approved practice guidelines about the management of uterine fibroids, including individual approach using different surgical methods, drug therapy and comparatively new methods like uterine artery embolization and focused energy delivery systems [4]. Some teams recommend new concepts in therapeutic management of symptomatic uterine fibroids that is 

patients in Europe, provided from experienced Chinese center-prospective comparative.

based on three pillars: surgery, pharmacotherapy, and interventional radiotherapy. They recommend HIFU as a noninvasive thermoablation of leiomyomas with minimum additional effects [5]. During the last decade, HIFU has been introduced as an innovative non-invasive method for thermal ablation of solid tumors. Different MRI- or US-guided devices have been used for the treatment of uterine fibroids over the last 15 years.

For diagnosis of tumors, HIFU therapy and assessment of the results of radiofrequency ablation, ultrasound contrast agents have been used. The microbubbles, which the contrast agents are made of, can make acoustic changes of the tissues and make HIFU treatment more efficient. There are many studies that show the use of contrast-enhanced HIFU treatment has effects and proved that SonoVue is safety and can be used in treatment of uterine fibroids to enhance the ablation effects of HIFU [6]. Most of them were performed in Asia. There are currently no studies that implement the Asian experience in Europe.

The purpose of this study is to determine whether the Chinese clinical protocol, ultrasound guided high-intensity focused ultrasound (USgHIFU) combined with real-time monitoring using a contrast-enhanced ultrasound (CEUS), also applies well in the European patients with uterine fibroids.

\section{Materials and Method}

\section{Patients}

This prospective study was approved by the ethics committee of Hospital A, Bulgaria and Hospital B, China. To be included in the study, each patient had to be examined by a gynecologist, a HIFU specialist, and have a contrast-enhanced MRI of the pelvis performed. On a special fibroid board, a final decision is made to include the patient in the study following the Patient Selection Criteria: (1) Clinically diagnosed fibroid; (2) Symptoms including an abnormal menstrual cycle, dysmenorrhea, secondary anemia, sterility; (3) $\mathrm{D} \geq 2.0 \mathrm{~cm}$ ( $\mathrm{D}>4 \mathrm{~cm}$-for a posterior wall fibroid); (4) Fibroid nodules visible on US; (5) Desire for HIFU therapy; (6) Desire for uterine preservation (refused hysterectomy); (7) Low and moderately vascularized fibroid nodules (T2 time of MRI to Hypo- to Medium intensed); (8) Less than 4 fibroid nodules; (9) Patient's BMI under 30; (10) The distance from the skin to the deepest point of the fibroid nodule less than $9 \mathrm{~cm}$.

Contraindications to include in the study: (1) Pregnancy; (2) Endometriosis; (3) Cervical fibroid; (4) Submucosal/subserosal fibroid on a pedicle; (5) $\mathrm{D} \leq 3.5 \mathrm{~cm}$ for the posterior wall fibroids; (6) Large postoperative abdominal wall scar; implanted foreign body along the acoustic path (not allowing US waves to pass through it); (7) Radiation with a dose over 45 Gray in the pelvis; (8) Hypovascularized fibroid nodule (hyperintense T2 time on MRI); (9) Subcutaneous fat above 5 $\mathrm{cm}$; (10) A malignant process such as uterine sarcoma, endometrial cancer, cervical cancer and others.
Clinical, laboratory and histological evaluation criteria were introduced for recognition of uterine sarcoma. In patients with hemoglobin below $70 \mathrm{~g} / \mathrm{L}$, rapidly growing fibroid nodules (over 1 year), LDH above $500 \mathrm{U} / \mathrm{L}$, heterointensed zones with liquid-equivalent zones, the patients were referred for surgical treatment.

Seventy-five (75) patients with 87 nodules of uterine fibroid from China and Bulgaria were treated with USgHIFU from April 2015 to July 2016, in the study. In Group A (Bulgarian) 26 (33 nodules) patients and in group B (Chinese) 49 (54 nodules) patients were included in the study (Table 1). All patients signed informed consent forms, and were able to voluntarily leave the study at any time.

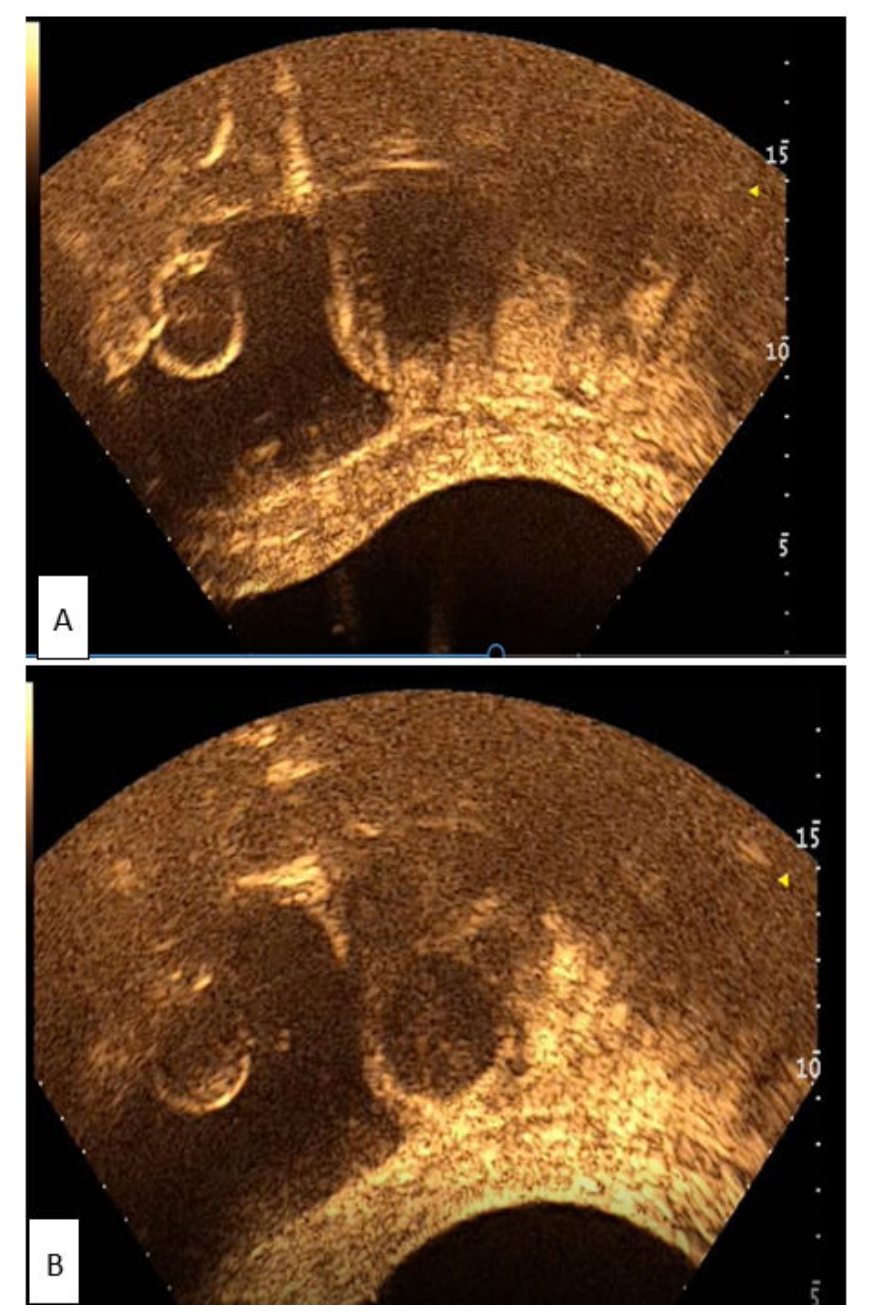

Figure 1. CEUS images of a 41 y old female patient with uterine fibroids pre-and post-HIFU. A) The red arrows point out the uterine fibroid enhanced with contrast agent during pre-HIFU CEUS. B) The yellow arrows point out the non-enhanced uterine fibroid post-HIFU with CEUS.

\section{Treatment protocols and HIFU procedures}

The real-time monitoring with CEUS was used in all the patients. The microbubble ultrasound contrast agent (SonoVue, Bracco, Milan, Italy) was dissolved at $25 \mathrm{mg}$ in $5 \mathrm{ml}$ normal saline and $1.5 \mathrm{ml}$ was administered intravenously before, during and after the USgHIFU ablation of the fibroids. After each 
dosage $10 \mathrm{ml}$ normal saline was injected i.v. The same clinical procedure of USgHIFU treatment as well as a follow-up was performed on patients from China and Bulgaria. The general characteristics of patients, the USgHIFU treatment and evaluation parameters, including complications, fibroid size, tumor volume, subcutaneous fat size, non-perfused volume
(NPV) and shrinkage process, were comparatively studied in Chinese and Bulgarian patients. Treatment time is defined as the period from the patient's arrival to the operating room (OR) until the patient leaves the OR. HIFU sonication time is defined as the period from the first test shot to the last treatment shot.

Table 1. Clinical characteristics of the patients with fibroid in the two groups.

\begin{tabular}{|c|c|c|c|}
\hline Clinical characteristics & Group A (Bulgarian patients) & Group B (Chinese patients) & Statistical data \\
\hline Age-mean \pm SD (years) & $41.8 \pm 5.07$ & $39.1 \pm 7.62$ & $t=-1.697, p=0.094$ \\
\hline Antero-posterior diameter (mm) & $51.7 \pm 22.93$ & $54.1 \pm 14.77$ & $\mathrm{t}=0.524, \mathrm{p}=0.603$ \\
\hline Left-right diameter (mm) & $52.3 \pm 22.04$ & $56.1 \pm 15.39$ & $\mathrm{t}=0.917, \mathrm{p}=0.362$ \\
\hline Vertical diameter (mm) & $55.5 \pm 26.16$ & $58.1 \pm 15.70$ & $\mathrm{t}=0.518, p=0.607$ \\
\hline pre-HIFU volume $\left(\mathrm{mm}^{3}\right)$ & $123780.2 \pm 150644.59$ & $108684.4 \pm 82225.03$ & $t=-0.529, p=0.599$ \\
\hline Fat size $(\mathrm{mm})$ & $17.1 \pm 8.58$ & $15.1 \pm 6.97$ & $t=-1.096, p=0.277$ \\
\hline Distance from skin to myoma (mm) & $105.2 \pm 20.67$ & $80.3 \pm 16.61$ & $t=-5.682, p=0,000$ \\
\hline
\end{tabular}

The evaluation of each patient was performed according to a contrast-enhanced MRI of abdominal organs made no more than 20 days prior to the procedure. The size, location, relationship with adjacent structures and organs and tumor morphology were evaluated. Each patient was subjected to a simulated exam with the HIFU apparatus in order to define under ultrasound control all anatomical orientations according to MRI images. The ultrasonic beam power was individually defined for each patient according to the anatomical structure, tumor site and size, cicatrix over the projection site of the tumor, thickness of the subcutaneous fold etc. Conventional biochemical tests, prothrombin time, full blood count, chest Xray, abdominal ultrasound and electrocardiography were performed and evaluated the day before ablation with HIFU. According to them, it was decided whether the patient would be under sedation during the procedure.

\section{Follow-Up and data collection}

All intraoperative data were recorded. The patients were discharged 1 day after HIFU treatment, and submitted to enhance MRI scanning up to 1 month after HIFU to determine non perfused volume (NPV). Finally, the patients were contacted the following 3 days to record post-procedure adverse events. The follow-up period was 12 months. Clinical examination and MRI were performed on the 6 th and $12^{\text {th }}$ month.

\section{Statistical analysis}

The SPSS software (SPSS 24, SPSS, Illinois, USA) was used for statistical analysis. Data were presented as median or mean \pm SD. The $\chi^{2}$ test was used. The Mann-Whitney test was utilized for comparison. Student's t test was used and $\mathrm{P}<0.05$ was considered statistically significant.

\section{Results}

\section{Clinical characteristics of the patients}

Table 1 summarizes the clinical characteristics of the patients. In group $\mathrm{A}$, the anterior-posterior diameter was $51.7 \pm 22.93$ $\mathrm{mm}$, the left-right size was $52.3 \pm 22.04 \mathrm{~mm}$, the vertical diameter was $55.5 \pm 26.16 \mathrm{~mm}$, the pre-HIFU volume of the fibroid nodules was $123780.2 \pm 150644.59 \mathrm{~mm}^{3}$, the fat size was $17.1 \pm 8.58 \mathrm{~mm}$ and the distance from skin to fibroid was $105.2 \pm 20.67 \mathrm{~mm}$. In group B, the anterior-posterior diameter was $54.1 \pm 17.77 \mathrm{~mm}$, the left-right size was $56.1 \pm 15.39 \mathrm{~mm}$, the vertical diameter was $58.1 \pm 15.70 \mathrm{~mm}$, the pre-HIFU volume of the fibroid nodules was $108684.4 \pm 82225.03 \mathrm{~mm}^{3}$, the fat size was $15.1 \pm 6.97 \mathrm{~mm}$ and the distance from skin to fibroid was $80.3 \pm 16.61 \mathrm{~mm}$. There were no significant differences in these parameters between the two groups $(\mathrm{P}>0.05)$.

\section{HIFU data}

All patients completed the HIFU treatment successfully in this study (Figure 1). Figure 1 shows the CEUS images pre-HIFU and post-HIFU during HIFU treatment protocol. Figure 1A shows enhancement of the uterine fibroid during CEUS preHIFU, while Figure 1B shows non-enhancement post-HIFU. In group A, the treatment time was $121.3 \pm 52.05 \mathrm{~min}$, the HIFU sonication time was $609.9 \pm 463.03 \mathrm{sec}$, the average power was $354.2 \pm 37.95 \mathrm{~W}$, the total energy was $205.2 \pm 144.42 \mathrm{KJ}$ and the HIFU sonication time per hour was $288.3 \pm 151.48 \mathrm{sec} / \mathrm{h}$. In group $\mathrm{B}$, the treatment time was $107.3 \pm 41.41 \mathrm{~min}$, the HIFU sonication time was $811.9 \pm 487.1 \mathrm{sec}$, the average power was $375.08 \pm 34.68 \mathrm{~W}$, the total energy was $309.3 \pm$ $194.48 \mathrm{KJ}$ and the HIFU sonication time per hour was $428.9 \pm$ $132.08 \mathrm{sec} / \mathrm{h}$ (Table 2 ). There were no significant differences in these parameters between the two groups $(\mathrm{P}>0.05)$. One case was lost from the Bulgarian group. 
Introducing clinical protocol for ultrasound-guided high-intensity focused ultrasound ablation of uterine fibroids in patients in Europe, provided from experienced Chinese center-prospective comparative.

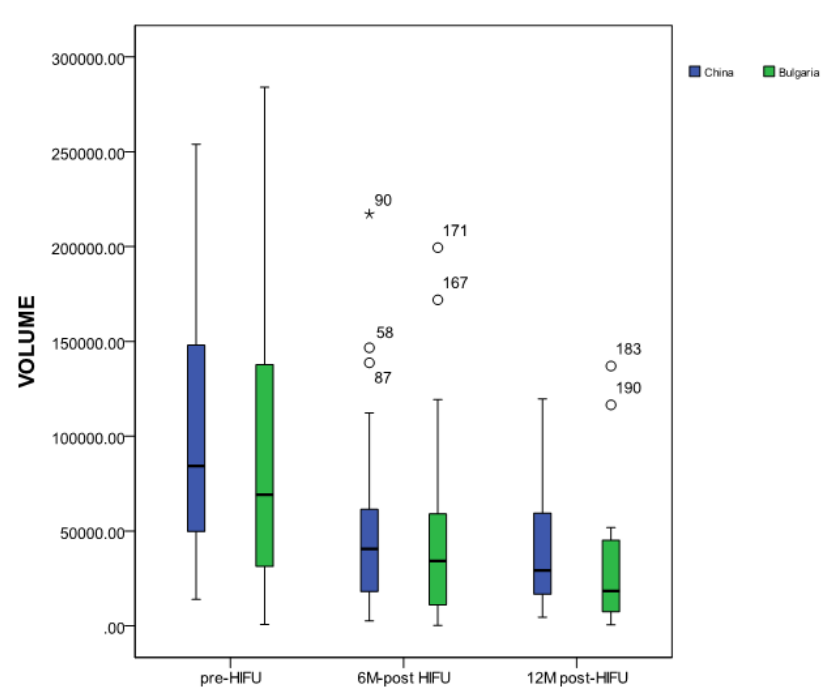

Figure 2. The comparison of the volumes at pre-HIFU, 6-months and 12-months post-HIFU in Bulgarian and Chinese patients.

Table 3 summarizes the results from T2-weighted images on MRI. There were no significant differences between the two groups $\left(\chi^{2}=1.680, \mathrm{P}=0.195\right)$.

Table 2. HIFU data.

\begin{tabular}{|c|c|c|c|c|c|c|}
\hline & Group & $\mathbf{N}$ & Mean & SD & $t$ & $P$ value \\
\hline \multirow{2}{*}{$\begin{array}{l}\text { Treatment } \\
\text { time (min) }\end{array}$} & $\begin{array}{l}\text { A (Bulgarian } \\
\text { patients) }\end{array}$ & 25 & 121.3 & 52.05 & \multirow{2}{*}{-1.257} & \multirow{2}{*}{0.213} \\
\hline & $\begin{array}{l}\text { B (Chinese } \\
\text { patients) }\end{array}$ & 49 & 107.3 & 41.41 & & \\
\hline \multirow{2}{*}{$\begin{array}{l}\text { HIFU } \\
\text { sonication } \\
\text { time (sec) }\end{array}$} & $\begin{array}{l}\text { A (Bulgarian } \\
\text { patients) }\end{array}$ & 25 & 609.9 & 463.03 & \multirow{2}{*}{1.715} & \multirow{2}{*}{0.091} \\
\hline & $\begin{array}{l}\text { B (Chinese } \\
\text { patients) }\end{array}$ & 49 & 811.9 & 487.1 & & \\
\hline \multirow{2}{*}{$\begin{array}{l}\text { Average } \\
\text { power }(\mathrm{W})\end{array}$} & $\begin{array}{l}\text { A (Bulgarian } \\
\text { patients) }\end{array}$ & 25 & 354.2 & 37.94 & \multirow{2}{*}{2.369} & \multirow{2}{*}{0.021} \\
\hline & $\begin{array}{l}\text { B (Chinese } \\
\text { patients) }\end{array}$ & 49 & 375.08 & 34.68 & & \\
\hline \multirow{2}{*}{$\begin{array}{l}\text { Total } \\
\text { energy (KJ) }\end{array}$} & $\begin{array}{l}\text { A (Bulgarian } \\
\text { patients) }\end{array}$ & 25 & 205.2 & 144.42 & \multirow{2}{*}{2.360} & \multirow{2}{*}{0.021} \\
\hline & $\begin{array}{l}\text { B (Chinese } \\
\text { patients) }\end{array}$ & 49 & 309.3 & 194.48 & & \\
\hline \multirow{2}{*}{$\begin{array}{l}\text { HIFU } \\
\text { sonication } \\
\text { time per } \\
\text { hour(sec/h) }\end{array}$} & $\begin{array}{l}\text { A (Bulgarian } \\
\text { patients) }\end{array}$ & 25 & 288.3 & 151.48 & \multirow{2}{*}{4.119} & \multirow{2}{*}{$<0.001$} \\
\hline & $\begin{array}{l}\text { B (Chinese } \\
\text { patients) }\end{array}$ & 49 & 428.9 & 132.08 & & \\
\hline
\end{tabular}

Table 3. Fibroid nodules contribution according to the T2-weighted magnetic resonance images (T2WI).

\begin{tabular}{lllll}
\hline Group & & Low signal & High signal & Total \\
\hline A (Bulgarian patients) & $\mathrm{N}$ & 13 & 20 & 33 \\
\cline { 2 - 5 } & $\%$ & $39.4 \%$ & $60.6 \%$ & $100.0 \%$ \\
\hline
\end{tabular}

\begin{tabular}{lllll}
\hline B (Chinese patients) & $\mathrm{N}$ & 29 & 25 & 54 \\
\cline { 2 - 5 } & $\%$ & $53.7 \%$ & $46.3 \%$ & $100.0 \%$ \\
\hline \multirow{2}{*}{ Total } & $\mathrm{N}$ & 42 & 45 & 87 \\
\cline { 2 - 5 } & $\%$ & $48.3 \%$ & $51.7 \%$ & $100.0 \%$ \\
\hline
\end{tabular}

Table 4. Comparison of the volumes at pre-HIFU, 6-months postHIFU and 12-months post-HIFU.

\begin{tabular}{|c|c|c|c|c|c|c|c|}
\hline & Group & $\begin{array}{l}\text { Numbe } \\
r \quad \text { of } \\
\text { cases } \\
\text { (n) }\end{array}$ & $\begin{array}{l}\text { Number } \\
\text { of } \\
\text { nodules } \\
\text { (n) }\end{array}$ & $\begin{array}{l}\text { Mean } \\
\text { volume } \\
\left(\mathrm{mm}^{3}\right)\end{array}$ & SD & $\mathbf{t}$ & $\begin{array}{l}P \\
\text { value }\end{array}$ \\
\hline \multirow{2}{*}{$\begin{array}{l}\text { Pre- } \\
\text { HIFU }\end{array}$} & $\begin{array}{l}\text { A } \\
\text { (Bulgarian } \\
\text { patients) }\end{array}$ & 26 & 33 & $\begin{array}{l}123780 . \\
2\end{array}$ & $\begin{array}{l}15064 \\
5\end{array}$ & \multirow{2}{*}{$\begin{array}{l}-0.52 \\
9\end{array}$} & \multirow{2}{*}{0.599} \\
\hline & $\begin{array}{l}\text { B } \\
\text { (Chinese } \\
\text { patients) }\end{array}$ & 49 & 54 & $\begin{array}{l}108684 . \\
4\end{array}$ & 82225 & & \\
\hline \multirow{2}{*}{$\begin{array}{l}6 \\
\text { months } \\
\text { post- } \\
\text { HIFU }\end{array}$} & $\begin{array}{l}\text { A } \\
\text { (Bulgarian } \\
\text { patients) }\end{array}$ & 22 & 26 & 48044.9 & $\begin{array}{l}52355 . \\
2\end{array}$ & \multirow{2}{*}{0.615} & \multirow{2}{*}{0.541} \\
\hline & $\begin{array}{l}\text { B } \\
\text { (Chinese } \\
\text { patients) }\end{array}$ & 36 & 37 & 57525.9 & $\begin{array}{l}65102 . \\
3\end{array}$ & & \\
\hline \multirow{2}{*}{$\begin{array}{l}12 \\
\text { months } \\
\text { post- } \\
\text { HIFU }\end{array}$} & $\begin{array}{l}\text { A } \\
\text { (Bulgarian } \\
\text { patients) }\end{array}$ & 13 & 13 & 34749.9 & $\begin{array}{l}43931 . \\
1\end{array}$ & \multirow{2}{*}{0.445} & \multirow{2}{*}{0.659} \\
\hline & $\begin{array}{l}\text { B } \\
\text { (Chinese } \\
\text { patients) }\end{array}$ & 25 & 27 & 40069.8 & $\begin{array}{l}30633 . \\
9\end{array}$ & & \\
\hline
\end{tabular}

\section{Comparison of the volumes at pre-HIFU, 6-months post-HIFU and 12-months post-HIFU}

In group A, the pre-HIFU volume was $123780.2 \pm 150644.59$ $\mathrm{mm}^{3}$, the 6-months post-HIFU volume was $48044.9 \pm$ $52355.19 \mathrm{~mm}^{3}$ and the 12 -months post-HIFU volume was $34749.9 \pm 43931.10 \mathrm{~mm}^{3}$. In group B, the pre-HIFU volume was $108684.4 \pm 82225.03 \mathrm{~mm}^{3}$, the 6 -months post-HIFU volume was $57525.9 \pm 65102.29 \mathrm{~mm}^{3}$ and the 12 -months postHIFU volume was $40069.8 \pm 30633.91 \mathrm{~mm}^{3}$ (Table 4). There were no significant differences in the volumes between the two groups at pre-HIFU, 6-months post-HIFU and 12-months postHIFU (Figure 2). Figure 2 shows the comparison of the volumes at pre-HIFU, 6-months and 12-months post-HIFU. At 6-months post-HIFU, 4 patients in the Bulgarian group were lost from follow-up and 9 more patients at 12-months postHIFU. In Chinese group, 13 patients were lost from follow up at 6-months post-HIFU and 9 more patients at 12-months postHIFU. The MR images of the same case of Figure 1 are in Figure 3. Figure $3 \mathrm{~A}$ shows the uterine fibroid pre-HIFU, Figure 3B shows that of 1-month post-HIFU and Figure 3C shows that of 6-months post-HIFU.

Comparison of the volumes between pre-HIFU and 6months post-HIFU, between pre-HIFU and 12- 


\section{months post-HIFU and between 6-months post-HIFU and 12-months post-HIFU in the Bulgarian group}

In group A there was a statistically significant volume reduction of the fibroid nodules at 6-months post-HIFU compared to the pre-HIFU volumes $(p=0.010)$ and a statistically significant volume reduction of the fibroid nodules at 12-months post-HIFU compared to the pre-HIFU volumes $(p=0.004)$. Significant differences between the volumes at 6months post-HIFU and at 12-months post-HIFU were not found $(\mathrm{p}=0.411)$ (Table 5).
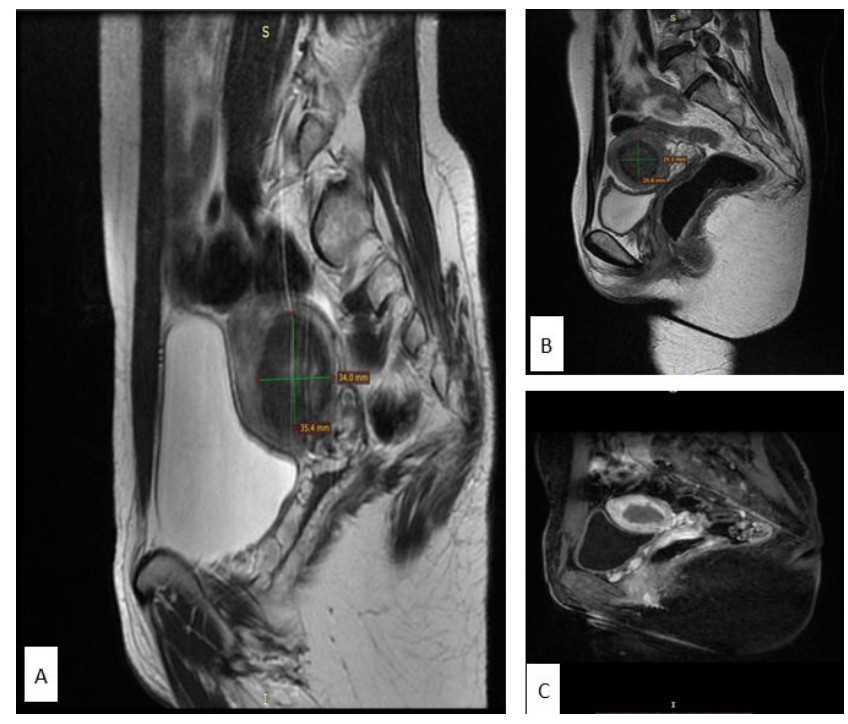

Figure 3. MR images of a 41 years old female patient with uterine fibroids pre-HIFU, 1 month and 6 month post-HIFU. A) preHIFUMR T2 image shows a intramural uterine fibroids with low signal with diameter $35.4 \mathrm{~mm} \times 34.0 \mathrm{~mm}$. B) 1 month post-HIFU MR T2 image shows the intramural uterine fibroids with diameter 29.8 $\mathrm{mm} \times 29.5 \mathrm{~mm}$. C) 6 month post-HIFU MR T2 image shows the intramural uterine fibroids with diameter less than $10 \mathrm{~mm}$.

\section{Comparison of the volumes between pre-HIFU and 6- months post-HIFU, between pre-HIFU and 12- months post-HIFU and between 6-months post-HIFU and 12-months post-HIFU in the Chinese group}

In group B there was a statistically significant volume reduction of the fibroid nodules at 6-months post-HIFU compared to the pre-HIFU volumes $(\mathrm{p}=0.002)$ and a statistically significant volume reduction of the fibroid nodules at 12-months post-HIFU compared to the pre-HIFU volumes $(p<0.05)$. Significant differences between the volumes at 6months post-HIFU and at 12-months post-HIFU were not found $(\mathrm{p}=0.202)$ (Table 6).

Table 5. Comparison of the volumes between pre-HIFU and 6-months post-HIFU, between pre-HIFU and 12-months post-HIFU and between 6-months post-HIFU and 12-months post-HIFU in the Bulgarian group.

\begin{tabular}{llll}
\hline & Pre-HIFU & $\begin{array}{l}\text { 6-months } \\
\text { HIFU }\end{array}$ & $\begin{array}{l}\text { post- } \\
\text { HIFU }\end{array}$ \\
\hline Pre-HIFU & $\mathrm{t}=2.689$ & $\mathrm{t}=3.079$ \\
\hline
\end{tabular}

\begin{tabular}{lll}
\cline { 3 - 3 } & $\mathrm{P}=0.010$ & $\mathrm{P}=0.004$ \\
\hline $\begin{array}{ll}\text { 6-months } \\
\text { HIFU }\end{array}$ & post- & $\mathrm{t}=0.786$ \\
\cline { 2 - 2 } & & $\mathrm{P}=0.411$ \\
\hline
\end{tabular}

Table 6. Comparison of the volumes between pre-HIFU and 6-months post-HIFU, between pre-HIFU and 12-months post-HIFU and between 6-months post-HIFU and 12-months post-HIFU in the Chinese group.

\begin{tabular}{|c|c|c|c|c|c|}
\hline & Pre-HIFU & $\begin{array}{l}\text { 6-months } \\
\text { HIFU }\end{array}$ & post- & $\begin{array}{l}\text { 12-months } \\
\text { HIFU }\end{array}$ & post- \\
\hline \multirow{2}{*}{\multicolumn{2}{|c|}{ Pre-HIFU }} & $t=3.164$ & & $\mathrm{t}=4.182$ & \\
\hline & & $P=0.002$ & & $P=0.000$ & \\
\hline \multirow{2}{*}{\multicolumn{2}{|c|}{ 6-months post-HIFU }} & & & $t=1.291$ & \\
\hline & & & & $P=0.202$ & \\
\hline
\end{tabular}

\section{Adverse events}

There were adverse events reported from patients during and post-HIFU treatment. Among those adverse events, lower abdominal pain was seen in 9 (Bulgarian group) vs. 15 (Chinese group) patients, sciatic pain or buttock pain in $3 \mathrm{vs}$. 10 patients and vaginal bleeding in 2 vs. 3 patients. There was no skin burn in both groups. No bowel injury or lumbosarcral nerve injury occurred. Adverse effects were naturally relieved within 1 week after HIFU treatment.

\section{Discussion}

HIFU treatment has been already included in the uterine fibroids treatment algorithm in Canada [4]. Studying uterine leiomyomas, Drayer et al mention that these energy systems are promising, but still not studied enough in European patients. For the assessment, more research and information is necessary [2]. A reduction of up to $98 \%$ in myoma volume and symptoms has been reported with MRI-guided HIFU for symptomatic myomas [7]. To increase the therapeutic efficiency of HIFU in achieving large ablation volume rapidly, various ultrasound contrast agents have been used with heating and cavitation effects, by modifying the acoustic environment in various tissues [8-15]. Among these, a phospholipid-shelled ultrasound contrast agent containing sulfur hexafluoride (SonoVue) is widely applied for diagnosis or evaluation after local ablation therapy in clinical settings, and assessed for enhancing the ablative effects of HIFU treatment $[11,16,17]$. Indeed, it was experimentally shown that SonoVue is effective in enhancing HIFU ablation in different tissues by enlarging the ablation volume [9], highly and rapidly increasing the temperature in the HIFU focal region $[10,18]$, or decreasing the total energy of HIFU, to a certain volume of ablation [8]. The results found in the literature demonstrated that SonoVue has heating and cavitation effects to enhance HIFU ablation without increasing the risk of adverse events.

A trial at China studying pregnancy outcomes in nulliparous women after ultrasound ablation of uterine fibroids including 
189 patients showed a pregnancy rate of $69.3 \%$ and a successful delivery rate of $76.3 \%$ [19]. Another study at China comparing HIFU, myomectomy and hysterectomy for treating symptomatic uterine fibroids showed that HIFU caused substantially less morbidity than surgery, with similar longterm QoL [20]. HIFU treatment seems to show equal quality in ablating uterine fibroids in patients with an anteverted uterus and the more difficult to treat retroverted uterus [21].

In order to evaluate the clinical application of HIFU treatment for European patients, we compared the efficacy and safety of patients from Bulgaria and China during April 2015 and July 2016. With the same protocol, we performed a total of seventyfive successful HIFU treatments. Clinical Characteristics of the Patients, HIFU parameters, the pre- and post-HIFU volumes of the two groups were compared. It was found that there is no statistically significant difference in age of the patients, size and volume of fibroid, size of subcutaneous fat tissue, Distance from skin to fibroid and MRI T2 signals of fibroid. Those data indicate the clinical basic situation of patients who were meeting the involved criteria were equal and comparable.

The parameters of HIFU treatment were compared too. The treatment time was $123.1 \pm 51.89 \mathrm{~min}$ in Bulgarian group vs. $105.8 \pm 42.26 \mathrm{~min}$ in Chinese group and with no statistically significant difference. The total HIFU sonication times in the groups were $609.9 \pm 463.03 \mathrm{sec}$ in Bulgarian group vs. 800.1 \pm 497.7 in Chinese group and with no statistically significant difference, either. Yet the Average power, Total energy and HIFU sonication time per hour in Bulgarian group were significantly lower than those in the Chinese group (375.8 \pm 33.91 W vs. $355.8 \pm 38.03 \mathrm{~W}, 305.0 \pm 198.21 \mathrm{KJ}$ vs. $205.2 \pm$ $144.42 \mathrm{KJ}, 425.3 \pm 136.66 \mathrm{sec} / \mathrm{h}$ vs. $288.3 \pm 151.48 \mathrm{sec} / \mathrm{h}$, separately). These data show that the Chinese group was with less HIFU treatment time but more HIFU energy delivery. As the Bulgarian HIFU team was with experience of 5 years while the Chinese HIFU with more than 15 years, we thought the HIFU clinical experience was the main factor which influenced the HIFU application and made the difference of HIFU data.

The volume comparisons among pre-HIFU, 6 months and 12 months post-HIFU indicated the efficacy of HIFU treatment of fibroid. Compared with that of pre-HIFU, volumes of myoma shrunk significantly at 6 months and 12 months post-HIFU in Bulgarian group and Chinese group, individually. In addition, the volumes of Bulgarian group and Chinese group were similar pre-HIFU; they were similar at 6 months post-HIFU, too; also similar at 12 months post-HIFU finally. This revealed the volume shrunk similarly in both groups at follow-up time points of 6 months and 12 months post-HIFU. That is the evidence that Chinese HIFU treatment protocol is efficient when used to treat Bulgarian patients.

The adverse events in both groups were reported with lower abdominal pain, sciatic pain or buttock pain and vaginal bleeding. During the first week post-HIFU, the mentioned adverse events were naturally relieved. No serious complications, such as skin burn, intestinal perforation or nerve injury, were recorded in this study. It revealed the introduction of Chinese HIFU treatment protocol is safe for Bulgarian patients, as well. There are weaknesses of this study. The number of patients is not as big as other clinical studies. The limitation was that we set the same involvement criteria in two different countries. Besides, we gathered these cases in 14 months, which was not a long period. Secondly, it will be better if we discuss other reasons explaining different HIFU data. We believe the clinical experience was the main one. Yet others, such as tolerance of pain or different social psychological situation, might influence the HIFU procedure. It will be studied in the near future.

\section{Conclusion}

To the best of our knowledge this is the first comparative study between the experiences of the Asian team and European center for USgHIFU treatment of uterine fibroid monitored by CEUS. The initial results of our study showed that CEUS clinical protocol for real time monitoring of efficacy and quality of the USgHIFU for uterine fibroid treatment that has been used in Chinese centers is efficient and safe when introduced in Bulgaria and may also be applicable for European patients. It could be used as a standard protocol for patients with fibroids both in Europe and China.

\section{Acknowledgment}

The study was sponsored by the Bulgarian Scientific fund (Research on the Potential of HIFU (high intensity focused ultrasound) Technology to treat Uterine Fibroids in Bulgarian and Chinese Patients as a Non-invasive Alternative Method to Conventional Surgery) and Chinese ministry of science and technology (clinical study of high intensity focused ultrasound ablation of uterine fibroids in Bulgaria and China, No. 141-14-2 international project of MOST).

\section{References}

1. Dzerov L. Benigh tumors of the uterus. In: Dimitrov A, Zlatkov V (Eds). Gynaecology (1st edn). Sofia: ARSO 2013; 2013: 225-227.

2. Drayer SM, Catherino WH. Prevalence, morbidity, and current medical management of uterine leiomyomas. Int $\mathrm{J}$ Gynecol Obstet 2015; 131: 117-122.

3. Duhan N. Current and emerging treatments for uterine myoma - an update. Int J Womens Health 2011; 3: 231-241.

4. Vilos GA, Allaire C, Laberge PY, Leyland N; SPECIAL CONTRIBUTORS. The management of uterine leiomyomas. J Obstet Gynaecol Can 2015; 37: 157-178.

5. Hoellen F, Bohlmann MK. New concepts in the therapeutic management of myoma. EMJ Repro Health 2015; 1: 87-94.

6. Cheng C, Xiao Z, Huang G, Zhang L, Baib J. Enhancing ablation effects of a microbubble contrast agent on highintensity focused ultrasound: an experimental and clinical study. BJOG 2017; 2017: 78-86.

7. de Melo FC, Diacoyannis L, Moll A, Tovar-Moll F. Reduction by $98 \%$ in uterine myoma volume associated 
with significant symptom relief after peripheral treatment with magnetic resonance imaging-guided focused ultrasound surgery. J Minim Invasive Gynecol 2009; 16: 501-503.

8. Tung YS1, Liu HL, Wu CC, Ju KC, Chen WS, Lin WL. Contrast-agent-enhanced ultrasound thermal ablation. Ultrasound Med Biol 2006; 32: 1103-1110.

9. Luo W, Zhou X, Yu M. Ablation of high-intensity fbcused ultrasound assisted with SonoVue on Rabbit VX2 liver tumors: sequential findings with histopathology, immunohistochemistry, andenzymehistochemistry. AnnSurg Oncol 2009; 16: 2359-2368.

10. Kaneko Y, Maruyama T, Takegami K, Watanabe T, Mitsui $\mathrm{H}$, Hanajiri $\mathrm{K}$, Nagawa $\mathrm{H}$, Matsumoto $\mathrm{Y}$. Use of a microbubble agent to increase the effects of high intensity focused ultrasound on liver tissue. Eur Radiol 2005; 15: 1415-1420.

11. Peng S, Xiong Y, Li K, He M, Deng Y, Chen L, Zou M, Chen W, Wang Z, He J, Zhang L. Clinical utility of a microbubble-enhancing contrast ("SonoVue") in treatment of uterine fibroids with high intensity focused ultrasound: a retrospective study. Eur J Radiol 2012; 81: 3832-3838.

12. Jiang N, Xie B, Zhang X. Enhancing ablation effects of a microbubbleenhancing contrast agent ("SonoVue") in the treatment of uterine fibroids with highintensity focused ultrasound: a randomized controlled trial. Cardiovasc Intervent Radiol 2014; 37: 1321-1328.

13. Moyer LC, Timbie KF, Sheeran PS. High-intensity focused ultrasound ablation enhancement in vivo via phase-shift nanodroplets compared to microbubbles. J Ther Ultrasound 2015; 3: 7.

14. Hamano N, Negishi Y, Takatori K. Combination of bubble liposomes and high-intensity focused ultrasound (HIFU) enhanced antitumor effect by tumor ablation. Biol Pharm Bull 2014; 37: 174-177.

15. Zhou D, Wang Z, Sun Y. Superparamagnetic PLGA-iron oxide microspheres as contrast agents for dual-imaging and enhance the effects of high intensity focused ultrasound ablation on liver tissue. Rsc Advances 2015; 5: 35693-35703.

16. Peng $\mathrm{S}, \mathrm{Hu} \mathrm{L}, \mathrm{Chen} \mathrm{W}$. Intraprocedure contrast enhanced ultrasound: the value in assessing the effect of ultrasoundguided high intensity focused ultrasound ablation for uterine fibroids. Ultrasonics 2015; 58: 123-128.

17. Wen YL, Kudo M, Zheng RQ, Minami Y, Chung H. Radiofrequency ablation of hepatocellular carcinoma: therapeutic response using contrast-enhanced coded phase-inversion harmonic sonography. AJR Am J Roentgenol 2003; 181: 57-63.

18. Dong JC, Lee JM, Hahn ST. Effect of microbubble contrast agent during high intensity focused ultrasound ablation on rabbit liver in vivo. Eur J Radiol 2012; 81: 519-523.

19. Li J, Wang Y, Chen J, Chen W. Pregnancy outcomes in nulliparous women after ultrasound ablation of uterine fibroids: A single-central retrospective study. Sci Rep 2017; 7: 3977.

20. Chen J, Li Y, Wang Z, McCulloch P, Hu L, Liu G, Li J, Lang J. Evaluation of high-intensity focused ultrasound ablation for uterine fibroids: an IDEAL prospective exploration study. BJOG 2017; 125: 354-364.

21. Zhang W, He M, Huang G, He J. A comparison of ultrasound-guided high intensity focused ultrasound for the treatment of uterine fibroids in patients with an anteverted uterus and a retroverted uterus. Int $\mathrm{J}$ Hyperthermia 2016; 32: 623-629.

\section{*Correspondence to}

Kun Zhou

Clinical Center for Tumor Therapy

The Second Affiliated Hospital

Chongqing Medical University

PR China 